very bad. The non-defective cases, with several vectors nearly parallel, were sometimes worse than the defective cases.

$N=6 \quad s=-4$

$\begin{array}{llcllllll}\text { actual roots } & 0, & 0 & 2, & 2 & -1 & -4 & -10 & -18 \\ \text { approximations (7070) } & .0228 & -.0225 & 1.995, & 2.004 & -1 & -4 & -10 & -18\end{array}$

$N=6 \quad s=-6$

$\begin{array}{lllll}\text { actual roots } & -6 & 0,0 & 4,4 & 6,6 \\ \text { approximations }(7070) & -6 & -.0056 \pm .111 i & 3.969 \pm .363 i & 6.036 \pm .2215 i\end{array}$

$N=6 \quad s=-6.5$

$\begin{array}{lllllll}\text { actual roots } & -3 & 0 & 2.5 & 4.5 & 6 & 7,7.75\end{array}$

$\begin{array}{lllllll}\text { approximations (7070) } & -3 & .0066 & 2.436 & 5.103 & 5.151 & 7.401 \pm .405 i\end{array}$

The effect of higher precision is seen in the following example which was run on both the 7070 (8 digits) and the 1604 (10 digits)

$N=10 \quad s=-14$

actual roots approximations (CDC 1604) approximations (IBM 7070)

$0 \quad-2 \times 10^{-7} \quad .0026$

$12 \quad 12.00004 \quad 11.898$

$22 \quad 21.995 \quad 20.195$

$\begin{array}{lll}30,30 & 29.638 \pm 1.134 i & 23.843 \pm 7.16 i\end{array}$

$36,36 \quad 34.993 \pm 2.94 i \quad 33.926 \pm 12.92 i$

$40,40 \quad 40.024 \pm 3.39 i \quad 36.977$

$42,42 \quad 43.347 \pm 1.49 i \quad 46.387 \pm 10.99 i$

52.594

University of Rochester

Rochester, New York

1. J. Brauner \& D. J. Wilson, "Intramolecular reactions II: A weak energy transfer mechanism," J. Phys. Chem., v. 67, 1963, p. 1134-1138.

2. P. J. Eberlein, "A Jacobi-like method for the automatic computation of eigenvalues and eigenvectors of an arbitrary matrix," J. Soc. Indust. Appl. Math., v. 10, 1962, p. 74-88.

\title{
Multivariate Polynomial Approximation for Equidistant Data
}

\section{By B. Mond}

Abstract. The theory of polynomial approximation for evenly spaced points is extended to multivariate polynomial approximation. It is also shown how available tables prepared for univariate approximation can be used in the multivariate case.

1. Introduction. Assume $f(x)$ is given for $x=x_{1}, x_{2}, \cdots, x_{n}$ and it is desired to approximate $f(x)$ by a polynomial of degree $p, 1 \leqq p<n$, i.e.

$$
f(x) \approx \sum_{i=0}^{p} a_{i} x^{i}
$$

Received November 12, 1963. 
In order to determine the coefficients $a_{i}$ so as to minimize

$$
\sum_{j=1}^{n}\left[f\left(x_{j}\right)-\sum_{i=0}^{p} a_{i} x_{j}{ }^{i}\right]^{2}
$$

one must solve a set of $p+1$ normal equations. If the $x_{i}$ are evenly spaced, the problem can be greatly simplified by a change of variable and the use of orthogonal polynomials [4]. This simplification will now be extended to approximation by multivariate polynomials.

2. Bivariate Polynomial Approximation. Assume that $f(x, y)$ is defined on a finite planar set of points $\left\{\left(x_{i}, y_{j}\right)\right\}(i=1,2, \cdots, n ; j=1,2, \cdots, m)$ and that it is desired to approximate $f(x, y)$ by a bivariate polynomial of the form

$$
\sum_{h=0}^{s} \sum_{k=0}^{r} a_{k h} x^{k} y^{h}
$$

$1 \leqq r<n$ and $1 \leqq s<m$. In order to determine the coefficients $a_{k h}(k=0,1, \cdots$, $r ; h=0,1, \cdots, s)$ so as to minimize

$$
\sum_{j=1}^{m} \sum_{i=1}^{n}\left[f\left(x_{i}, y_{j}\right)-\sum_{h=0}^{s} \sum_{k=0}^{r} a_{k h} x_{i}{ }^{k} y_{j}\right]^{2}
$$

one must solve a system of $(r+1)(s+1)$ normal equations.

Assume, now, that the $x_{i}$ and $y_{j}$ are evenly spaced, i.e.

$$
\begin{array}{ll}
x_{k+1}-x_{k}=h_{1} & (k=1,2, \cdots, n-1) \\
y_{k+1}-y_{k}=h_{2} & (k=1,2, \cdots, m-1) .
\end{array}
$$

Thus, if we write

$$
\begin{aligned}
& x=a+i h_{1} \\
& y=b+j h_{2},
\end{aligned}
$$

where $a=x_{1}-h_{1}$ and $b=y_{1}-h_{2},(x, y)$ will represent the coordinates of the given points when $i=1, \cdots, n ; j=1, \cdots, m$. Let $\bar{x}$ and $\bar{y}$ be the midpoints of the given $x_{i}$ and $y_{j}\left[\bar{x}=\left(x_{1}+x_{n}\right) / 2 ; \bar{y}=\left(y_{1}+y_{m}\right) / 2\right]$. The horizontal and vertical distances from the midpoint divided by the uniform spacing $\left[(x-\bar{x}) / h_{1}\right.$ and $\left.(y-\bar{y}) / h_{2}\right]$ are then equal respectively, by virtue of equations (1), to $i-(n+1) / 2$ and $j-(m+1) / 2$.

Let $P_{h k}(h=0,1, \cdots, r ; k=0,1, \cdots, s)$ be a set of polynomials of exact degree $r$ and $s$ in $i-(n+1) / 2$ and $j-(m+1) / 2$, i. e.

$$
\begin{aligned}
P_{h k}=\alpha_{00}^{h k}+ & \alpha_{10}^{h k}[i-(n+1) / 2]+\alpha_{01}^{h k}[j-(m+1) / 2] \\
& +\alpha_{11}^{h k}[i-(n+1) / 2][j-(m+1) / 2]+\cdots \\
& +\alpha_{h k}^{h k}[i-(n+1) / 2]^{h}[j-(m+1) / 2]^{k} .
\end{aligned}
$$

$P_{00}$ will always be taken equal to one.

If, now, we approximate $f(x, y)$ by a polynomial of the form

$$
\sum_{h=0}^{s} \sum_{k=0}^{r} b_{k h} P_{k h}
$$


and solve for the constants $b_{k n}$ in the sense of least squares, we obtain $(r+1)$ $(s+1)$ normal equations with the augmented matrix

$$
\left[\begin{array}{ccccc}
\sum \sum P_{00} & \sum \sum P_{01} \cdots \sum \sum P_{0 s} & \sum \sum P_{10} \cdots \sum \sum P_{1 s} \cdots \sum \sum P_{r s} & \sum \sum f\left(x_{i}, y_{j}\right) \\
\sum \sum P_{00} P_{01} & \sum \sum P_{01}^{2} \cdots \sum \sum P_{0 s} P_{01} \cdots & \sum \sum P_{r s} P_{01} & \sum \sum f\left(x_{i}, y_{j}\right) P_{01} \\
\vdots & \vdots & & \\
\sum \sum P_{00} P_{0 s} & \sum \sum P_{01} P_{0 s} \cdots \sum \sum P_{0 s}^{2} \cdots & \sum \sum P_{r s} P_{0 s} & \sum \sum f\left(x_{i}, y_{j}\right) P_{0 s} \\
\sum \sum P_{00} P_{r s} & \sum \sum P_{01} P_{r s} \cdots \sum \sum P_{0 s} P_{r s} \cdots & \sum \sum P_{r s}^{2} & \sum \sum f\left(x_{i}, y_{j}\right) P_{r s}
\end{array}\right]
$$

where the notation $\sum \sum P_{h k} P_{c d}$ means

$$
\sum_{j=1}^{m} \sum_{i=1}^{n} P_{h k}[i-(n+1) / 2, j-(m+1) / 2] P_{c d}[i-(n+1) / 2, j-(m+1) / 2] \text {. }
$$

If the $\alpha_{i j}^{h k}$ in (2) are chosen so that the polynomials $P_{h k}$ are biorthogonal, i.e.

$$
\sum_{j=1}^{m} \sum_{i=1}^{n} P_{h k} P_{c d}=0 \quad \text { if } h \neq c \text { or } k \neq d,
$$

the coefficient matrix reduces to a diagonal matrix. The $b_{h k}$ can then be written as

$$
b_{h k}=\sum_{j} \sum_{i} f\left(x_{i}, y_{j}\right) P_{h k} / \sum_{j} \sum_{i} P_{h k}^{2} .
$$

As in the univariate case [2], testing the appropriatness of the representation is also facilitated by the use of orthogonal polynomials. Should a polynomial of higher degree be desired, the coefficients $b_{h k}$ need not be recalculated.

3. Constructing Biorthogonal Polynomial Tables. In approximating with orthogonal functions, many of the calculations necessary are independent of the data [for example, the denominator in (3)] and need not be recalculated each time a different set of data is to be fitted. Extensive tables are available for univariate orthogonal polynomials [1], [2]. Their use makes the actual calculation of the coefficients quite easy.

By appropriately choosing the bivariate biorthogonal polynomials, univariate tables that are already available can easily be modified for use in the bivariate case.

Theorem. Let $P_{h}(h=0,1, \cdots, r)$ and $P_{k}(k=0,1, \cdots, s)$ be sets of orthogonal polynomials in $(i-(n+1) / 2)$ and $(j-(m+1) / 2)$ respectively with $P_{0}=1$. Let $P_{h k}=P_{h} P_{k} . P_{h k}(h=0,1, \cdots, r ; k=0,1, \cdots, s)$ is then a set of bivariate biorthogonal polynomials in $(i-(n+1) / 2)$ and $(j-(m+1) / 2)$.

Proof. It follows from the definition that $P_{h k}$ will be a bivariate polynomial of degree $h$ and $k$ in $(i-(n+1) / 2)$ and $(j-(m+1) / 2)$ respectively.

Biorthogonality of the bivariate polynomials follows from the orthogonality of the univariate polynomials and the fact that

$$
\sum_{j} \sum_{i} P_{h k} P_{c d}=\sum_{j} \sum_{i} P_{h} P_{k} P_{c} P_{d}=\sum_{j} P_{k} P_{c} \sum_{i} P_{h} P_{d} .
$$

Taking $P_{h k}=P_{h} P_{k}$, it is possible to utilize, in bivariate polynomial approximation, tables that were constructed for use in the univariate case. In general, if one regards the rectangular array of values for a given $n$ in Fisher and Yates statistical tables [2] as a matrix, then the corresponding matrix for bivariate biorthogonal polynomials would be a Kronecker product [5] of corresponding matrices. 
For example, from the entries for $n=3$ and $n=4$ in the Fisher and Yates tables [2], one gets as the entry in the bivariate table for $n=3, m=4$

\begin{tabular}{|c|c|c|c|c|c|c|c|c|c|c|c|c|}
\hline$\imath$ & $j$ & $P_{01}$ & $P_{02}$ & $P_{03}$ & $P_{10}$ & $P_{11}$ & $P_{12}$ & $P_{13}$ & $P_{20}$ & $P_{21}$ & $P_{22}$ & $P_{23}$ \\
\hline 1 & 1 & -3 & +1 & -1 & -1 & +3 & -1 & +1 & +1 & -3 & +1 & -1 \\
\hline 1 & 2 & -1 & -1 & +3 & -1 & +1 & +1 & -3 & +1 & -1 & -1 & +3 \\
\hline 1 & 3 & +1 & -1 & -3 & -1 & -1 & +1 & +3 & +1 & +1 & -1 & -3 \\
\hline 1 & 4 & +3 & +1 & +1 & -1 & -3 & -1 & -1 & +1 & +3 & +1 & +1 \\
\hline 2 & 1 & -3 & +1 & -1 & 0 & 0 & 0 & 0 & -2 & +6 & -2 & +2 \\
\hline 2 & 2 & -1 & -1 & +3 & 0 & 0 & 0 & 0 & $-\overline{2}$ & +2 & +2 & -6 \\
\hline 2 & 3 & +1 & -1 & -3 & 0 & 0 & 0 & 0 & -2 & -2 & +2 & +6 \\
\hline 2 & 4 & +3 & +1 & +1 & 0 & 0 & 0 & 0 & -2 & -6 & -2 & -2 \\
\hline 3 & 1 & -3 & +1 & -1 & +1 & -3 & +1 & -1 & +1 & -3 & +1 & -1 \\
\hline 3 & 2 & -1 & -1 & +3 & +1 & -1 & -1 & +3 & +1 & -1 & -1 & +3 \\
\hline 3 & 3 & +1 & -1 & -3 & +1 & +1 & -1 & -3 & +1 & +1 & -1 & -3 \\
\hline \multirow[t]{2}{*}{3} & \multirow[t]{2}{*}{4} & +3 & +1 & +1 & +1 & +3 & +1 & +1 & +1 & +3 & +1 & +1 \\
\hline & & 60 & 12 & 60 & 8 & 40 & 8 & 40 & 24 & 120 & 24 & 120 \\
\hline
\end{tabular}

where the last line represents the sums of squares of all elements in the particular column. All entries in the bivariate table are products of corresponding entries in the univariate table.

Bivariate tables constructed as outlined here could be used in exactly the same manner as recommended for univariate tables [see 2].

4. Extension to $n$ Variables. The extension to $n$ variables is straightforward. The $n$ dimension analogue to equation (3) is

$$
b_{h_{1}} \cdots h_{n}=\sum_{i_{n}=1}^{m_{n}} \ldots \sum_{i_{1}=1}^{m_{1}} f\left(x_{i_{1}}^{(1)}, x_{i_{2}}^{(2)}, \cdots, x_{i_{n}}^{(n)}\right) P_{h_{1} \cdots h_{n}} / \sum_{i_{n}=1}^{m_{n}} \ldots \sum_{i_{1}=1}^{m_{1}} P_{h_{1}}^{2} \cdots h_{n}
$$

where the $P_{h_{1} \ldots h_{n}}$ are orthogonal polynomials in $n$ variables and the $b_{h_{1} \cdots h_{n}}$ are the coefficients in the approximation

$$
f\left(x^{(1)}, \cdots, x^{(n)}\right) \approx \sum_{h_{n}=0}^{r_{n}} \cdots \sum_{h_{1}=0}^{r_{1}} b_{h_{1} \ldots h_{n}} P_{h_{1}} \cdots h_{n} / \sum_{i_{n}=1}^{m n} \cdots \sum_{i_{1}=1}^{m_{1}} P_{h_{1} \cdots h_{n}}^{2}
$$

so as to minimize

$$
\sum_{i_{n}=1}^{m_{n}} \cdots \sum_{i_{1}=1}^{m_{1}}\left[f\left(x_{i_{1}}^{(1)}, \cdots, x_{i_{n}}^{(n)}\right)-\sum_{h_{n}=0}^{r_{n}} \cdots \sum_{h_{1}=0}^{r_{1}} b_{h_{1} \cdots h_{n}} P_{h_{1} \cdots h_{n}}\right]^{2}
$$

As in the bivariate case, one can make use of univariate tables in multivariate polynomial approximation by taking for the multivariate polynomials the products of corresponding univariate orthogonal polynomials.

Aerospace Research Laboratories

Wright-Patterson Air Force Base, Ohio

1. R. L. Anderson \& E. E. Houseman, "Tables of orthogonal polynomial values extended to $n=104$," Research Bulletin 292, Ames, Iowa, 1942.

2. R. A. Fisher \& F. Yates, Statistical Tables for Biological, Agricultural and Medical Research, Hafner Publishing Company, New York, 1957.

3. F. A. Graybill, An Introduction to Linear Statistical Models, Vol. 1, McGraw Hill Company Inc., New York, 1961.

4. F. B. Hildebrand, Introduction to Numerical Analysis, McGraw Hill Company Inc., New York, 1956.

5. C. C. MacDuffee, Theory of Matrices, Chelsea Publishing Company, New York, 1936. 